\title{
Understanding the nature of FR II optical nuclei: A new diagnostic plane for radio galaxies $\star$
}

\author{
M. Chiaberge $\mathrm{e}^{1,2, \star \star}$, A. Capetti ${ }^{3}$, and A. Celotti ${ }^{4}$ \\ 1 Space Telescope Science Institute, 3700 San Martin Drive, Baltimore, MD 21218, USA \\ 2 Instituto di Radioastronomia del CNR, Via P. Gobetti 101, 40129 Bologna, Italy \\ 3 Instituto Nazionale di Astrofisica, Osservatorio Astronomico di Torino, Strada Osservatorio 20, 10025 Pino Torinese, Torino, \\ Italy \\ 4 SISSA/ISAS, Via Beirut 2-4, 34014 Trieste, Italy
}

Received 30 November 2001 / Accepted 28 June 2002

\begin{abstract}
We extend our study of the nuclei of 3CR FR II radio galaxies through HST optical images up to $z=0.3$. In the majority of them an unresolved nucleus (central compact core, $\mathrm{CCC}$ ) is found. We analyze their position in the plane formed by the radio and optical nuclear luminosities in relation to their optical spectral properties. The broad-lined objects (BLO) have the brightest nuclei: they are present only at optical luminosities $v L_{v} \approx 4 \times 10^{42} \mathrm{erg} \mathrm{s}^{-1}$ which we suggest might represent a threshold in the radiative efficiency combined with a small range of black hole masses. About $40 \%$ of the high and low excitation galaxies (HEG and LEG) show CCC which resemble those previously detected in FR I galaxies, in apparent contrast to the unification model. The equivalent width of the [OIII] emission line (with respect to the nuclear luminosity) reveals the nature of these nuclei, indicating that the nuclei of HEG are obscured to our line of sight and only scattered radiation is observed. This implies that the population of FR II is composed of objects with different nuclear properties, and only a fraction of them can be unified with quasars.
\end{abstract}

Key words. galaxies: active - galaxies: nuclei - galaxies: jets - quasars: general

\section{Introduction}

In the framework of the AGN unification scheme for radioloud sources, powerful radio galaxies with FR II edgebrightened morphology (Fanaroff \& Riley 1974) are believed to be misaligned quasars, while lower power, edge-darkened FR I are associated with BL Lac objects. This basic unification picture (Barthel 1989; Orr \& Browne 1982, see Urry \& Padovani 1995 for a review) is mainly supported by the comparison of the extended properties (radio morphology and linear dimensions, host galaxy type and environment, and narrow emission line luminosity) as well as by the number counts of the two classes. However, the detection of polarized broad emission lines, although only for a small number of FR II, is the most direct evidence of the unification scheme and the presence of absorbing "tori" in high power radio loud sources (Antonucci \& Barvainis 1990; Cohen et al. 1999).

Send offprint requests to: M. Chiaberge, e-mail: chiab@stsci.edu

* Based on observations obtained at the Space Telescope Science Institute, which is operated by the Association of Universities for Research in Astronomy, Incorporated, under NASA contract NAS 5-26555.

$\star \star$ ESA fellow.
How these results extend to low power sources is still unclear. In fact, on large scales, the morphological FR I/FR II dicothomy appears to be also associated with other (large scale) properties. From the optical point of view, FR II are associated with different sub-classes of bright elliptical galaxies. On average, FR II hosts are less luminous with respect to FR I ones (Owen 1993; Ledlow \& Owen 1996), and belong to lower density groups, at least at low redshifts (e.g. Zirbel 1996, 1997).

However, how these large-scale properties relate to the nuclear structure and central activity is still a debated issue. In particular, the properties of the emission lines observed in the spectrum of radio galaxies and plausibly connected to the nuclear activity, have revealed a phenomenology richer than the radio one. For example, the role of a sub-class of low-ionization FR II (e.g. Laing et al. 1994) has still to be assessed. These objects have an FR II morphology, but their optical spectral properties are similar to those of FR I. Wall \& Jackson (1997) and Jackson \& Wall (1999) proposed that such objects constitute, together with FR I, a single population of radio galaxies. Furthermore, Willott et al. (2000) have recently found that the fraction of objects with observed broad lines (in the $6 \mathrm{C}$, 7C and 3CRR samples and having excluded FR I) decreases with luminosity. They indicate as a possible explanation for 
this lack of quasars the rise of a distinct population of radio galaxies which have an FR II radio morphology but lack a wellfed quasar nucleus.

Our aim is to investigate these issues by directly looking at the nuclear continuum emission in the optical band, identifying its physical origin and relating it to both the radio and emission line properties. HST optical images of the nuclear regions of radio galaxies are suited to this goal as their high resolution allows us to separate the AGN emission from the stellar host galaxy background. In particular, the optical snapshot surveys of 3CR objects (e.g. Martel et al. 1999; De Koff et al. 1996) has provided a wealth of high quality data for this purpose.

We have started this study by considering complete samples of FR I and FR II from this catalog (Chiaberge et al. 1999, hereafter Paper I; Chiaberge et al. 2000, hereafter Paper II). We analyzed the properties of unresolved optical nuclei, which have been found to be present in the great majority of the objects.

The optical nuclei of 3CR FR I behave similarly, and are best explained as non thermal synchrotron emission from the base of the relativistic jet. Furthermore, the high detection rate $(\sim 85 \%)$ directly implies that geometrically thick obscuring tori are not present in FR I radio galaxies (or, alternatively, they are present only in a minority of them). Given this, the lack of broad emission lines in these objects cannot be due to obscuration.

The behavior of FR II (at redshift below $z=0.1$ ) appears to be more complex, although their properties are clearly related to their spectral classification. Broad line radio galaxies show an optical excess with respect to the expected non thermal emission level, which might indicate a contribution from the thermal disk. Several radio galaxies in which broad lines are absent do not show any nuclear source, and they can be interpreted as obscured nuclei, as expected in the framework of the current AGN unification scheme. Most importantly, 5 sources of the sample have a core with radio-optical properties that are completely consistent with those found in FR I. Although not all of them belong to the low-ionization subclass, it is tempting to consider them as FR I-like.

In all cases, this nuclear emission sets an upper limit to any radiation from the accretion flow. In particular, in the nonthermally dominated nuclei (FR I or FR II) this seems to imply that accretion might take place on a low efficiency radiative regime.

Since it is important both to establish whether these findings hold only for nearby sources, and to analyze a larger sample of objects in order to improve the statistics, in this paper we extend the sample up to a redshift of $z=0.3$. Furthermore we will show that in order to address the nature of the nuclei, a crucial parameter is the equivalent width of the [OIII] emission line, which we calculate with respect to the nuclear continuum emission. The organization of the paper is as follows: in Sect. 2 we describe our sample of FR II and the HST observations; in Sect. 3 we present the results of the photometry of the nuclei and we analyze the relation between the optical and radio core luminosity; in Sect. 4 we discuss our results for the different spectral subclasses, also considering their radio properties and, most importantly, the [OIII] emission line luminosity. In Sect. 5 we present a summary of our findings and we draw conclusions and future perspectives.

$H_{0}=75 \mathrm{~km} \mathrm{~s}^{-1} \mathrm{Mpc}^{-1}$ and $q_{0}=0.5$ are adopted throughout the paper. The spectral index $\alpha$ is defined as $F_{v} \propto v^{-\alpha}$.

\section{The sample and the HST observations}

The sample considered here comprises all radio galaxies belonging to the 3CR catalogue (Spinrad 1985) with redshift $z<$ 0.3 , morphologically classified as FR II. We directly checked their classification for erroneous or doubtful identifications by searching the literature for the most recent radio maps. The final list constitutes a complete, flux and redshift limited sample of 65 FR II radio galaxies.

We searched for optical spectral classification and/or optical spectra, in order to differentiate our sources on the basis of the presence of broad or narrow and high or low excitation emission lines. Here we adopt the scheme defined by Jackson \& Rawlings (1997). They classify as WQ (weak quasar) objects for which at least one broad line has been observed and whose optical continuum $V$-band luminosity is $<10^{23} \mathrm{~W} \mathrm{~Hz}^{-1}$ while quasars (QSO) have brighter continua; high and low ionization narrow-lined galaxies (HEG and LEG) are classified on the basis of their [OIII] equivalent width (less than $10 \AA$ for a LEG) and/or [OII]/[OIII] ratio (>1 for a LEG). In the following, we will also refer to WQ and QSO as the broad-lined objects (BLO).

In Table 1 we show redshifts and radio data of objects with $z<0.3$, as taken from the literature, together with the optical spectral classifications. Low redshift objects $(z<0.1)$ have been already discussed in Paper II and are listed here for completeness.

In Fig. 1 we show the redshift vs total radio luminosity diagram for the sample of FR II galaxies, together with the sample of FR I discussed in Paper I. FR II have a median redshift $z=0.152$, and total radio luminosities at $178 \mathrm{MHz}$ are between $10^{32}$ and $10^{34.4} \mathrm{erg} \mathrm{s}^{-1} \mathrm{~Hz}^{-1}$. Note that whereas the two samples are selected at the same limits of redshift and flux, FR II are, on average, more luminous and distant than FR I. Furthermore, there is no significant difference in the distributions of extended radio power for the different spectral classes of FR II.

HST observations were performed during the 3CR snapshot survey (Martel et al. 1999; De Koff et al. 1996) and are available in the public archive for all 65 objects except 4 (3C 33, 3C 61.1 and 3C 105, 3C 458 which were not observed). The whole sample was observed using the $F 702 \mathrm{~W}$ filter except for 3C $192(F 555 W)$. Exposure times are in the range 140-300 s. The data were processed through the standard PODPS (Post Observation Data Processing System) pipeline for bias removal and flat fielding (Biretta et al. 1996). Individual exposures in each filter were combined to remove cosmic rays events.

\section{Optical cores in FR II and their relationship with radio cores}

We derive the radial brightness profiles of all objects, looking for unresolved nuclear components (central compact 
Table 1. The sample of FR II radiogalaxies.

\begin{tabular}{|c|c|c|c|c|c|c|c|c|c|c|c|}
\hline Source Name & $\begin{array}{c}\text { redshift } \\
z\end{array}$ & Class. & $\begin{array}{c}S_{\mathrm{t}}(178) \\
\mathrm{Jy}\end{array}$ & $\begin{array}{c}\log L_{\mathrm{r}} \\
\operatorname{erg~s}^{-1} \mathrm{~Hz}^{-1}\end{array}$ & $\underset{\mathrm{erg} \mathrm{s}^{-1}}{\log L_{[\mathrm{OIII}}}$ & Source Name & $\begin{array}{c}\text { redshift } \\
z\end{array}$ & Class. & $\begin{array}{c}S_{\mathrm{t}}(178) \\
\mathrm{Jy}\end{array}$ & $\begin{array}{c}\log L_{\mathrm{r}} \\
\operatorname{erg~s}^{-1} \mathrm{~Hz}^{-1}\end{array}$ & $\begin{array}{c}\log L_{[\mathrm{OIII}]} \\
\mathrm{erg} \mathrm{s}^{-1}\end{array}$ \\
\hline $3 \mathrm{C} 15$ & 0.073 & LEG & 15.8 & 31.54 & 40.38 & $3 \mathrm{C} 227^{a}$ & 0.086 & WQ & 30.0 & 30.48 & 41.78 \\
\hline $3 \mathrm{C} 17$ & 0.220 & WQ & 20.0 & 32.76 & 41.91 & $3 \mathrm{C} 234$ & 0.185 & WQ & 26.1 & 31.88 & 43.17 \\
\hline $3 \mathrm{C} 18$ & 0.188 & HEG & 19.8 & 31.84 & 41.88 & $3 \mathrm{C} 236$ & 0.099 & LEG & 20.5 & 31.51 & 40.47 \\
\hline $3 \mathrm{C} 33$ & 0.059 & HEG & 53.0 & 30.27 & 42.01 & 3C 284 & 0.239 & HEG & 6.1 & 30.25 & 42.19 \\
\hline 3C 33.1 & 0.181 & WQ & 11.4 & 31.03 & 41.66 & 3C 285 & 0.079 & HEG & 6.0 & 29.93 & 40.73 \\
\hline 3C 35 & 0.067 & LEG & 7.3 & 30.27 & 40.03 & 3C 287.1 & 0.216 & QSO & 14.2 & 32.53 & - \\
\hline 3C 40 & 0.018 & LEG & 28.6 & 30.60 & 38.85 & 3C 300 & 0.270 & HEG & 17.97 & 31.07 & 42.16 \\
\hline $3 \mathrm{C} 61.1$ & 0.184 & LEG & 34.51 & 30.33 & 42.09 & 3C 303 & 0.141 & WQ & 10.6 & 31.80 & - \\
\hline $3 \mathrm{C} 63$ & 0.175 & HEG & 19.1 & 30.97 & - & 3C 318.1 & 0.046 & LEG & 12.0 & - & - \\
\hline 3C 79 & 0.256 & HEG & 24.8 & 31.19 & 42.43 & 3C 319 & 0.192 & LEG & 14.2 & 29.93 & - \\
\hline $3 \mathrm{C} 88$ & 0.030 & LEG & 17.5 & 30.50 & 39.78 & 3C 321 & 0.096 & HEG & 11.2 & 30.78 & 42.15 \\
\hline 3С 98 & 0.030 & HEG & 35.5 & 29.25 & 40.87 & $3 \mathrm{C} 323.1$ & 0.264 & QSO & 10.0 & 31.69 & 42.93 \\
\hline $3 \mathrm{C} 105$ & 0.089 & HEG & 18.0 & 30.36 & 40.78 & $3 \mathrm{C} 326$ & 0.089 & LEG & 7.8 & 30.34 & 40.64 \\
\hline $3 \mathrm{C} 111$ & 0.049 & WQ & 35.2 & 31.69 & - & $3 \mathrm{C} 327$ & 0.104 & HEG & 43.0 & 30.88 & 42.05 \\
\hline $3 \mathrm{C} 123$ & 0.218 & LEG & 189.0 & 31.82 & 41.58 & 3C 332 & 0.152 & QSO & 9.5 & 30.65 & - \\
\hline $3 \mathrm{C} 132$ & 0.214 & LEG & 12.4 & 31.40 & - & 3C 349 & 0.205 & HEG & 13.3 & 31.18 & 41.42 \\
\hline 3C 133 & 0.277 & - & 21.0 & 32.32 & - & $3 \mathrm{C} 353$ & 0.030 & LEG & 220.0 & 30.54 & 39.34 \\
\hline 3C 135 & 0.127 & HEG & 18.2 & 30.18 & - & 3C 357 & 0.167 & HEG & 8.2 & 30.48 & - \\
\hline 3C 136.1 & 0.064 & - & 14.0 & - & - & 3C 379.1 & 0.256 & HEG & - & - & - \\
\hline 3C 153 & 0.277 & HEG & 16.67 & 29.73 & - & $3 \mathrm{C} 381$ & 0.161 & HEG & 15.2 & 30.48 & 42.37 \\
\hline $3 \mathrm{C} 165$ & 0.296 & LEG & 13.5 & 31.08 & - & 3C 382 & 0.058 & WQ & 15.2 & 31.13 & 41.52 \\
\hline $3 \mathrm{C} 166$ & 0.245 & - & 14.7 & 32.73 & - & $3 \mathrm{C} 388^{b}$ & 0.091 & LEG & 22.5 & 31.04 & 40.49 \\
\hline $3 \mathrm{C} 171$ & 0.238 & HEG & 21.8 & 30.36 & 42.50 & $3 \mathrm{C} 390.3^{b}$ & 0.056 & WQ & 52.4 & 31.38 & 42.05 \\
\hline 3C 173.1 & 0.292 & LEG & 14.3 & 31.18 & 41.35 & $3 \mathrm{C} 401$ & 0.201 & LEG & 24.0 & 31.50 & 41.01 \\
\hline 3C 180 & 0.220 & - & 14.2 & - & - & $3 \mathrm{C} 402$ & 0.025 & HEG & 9.0 & 29.73 & - \\
\hline 3C 184.1 & 0.118 & HEG & 14.3 & 30.25 & 42.19 & $3 \mathrm{C} 403$ & 0.059 & HEG & 28.0 & 29.87 & 41.55 \\
\hline 3C 192 & 0.060 & HEG & 23.2 & 29.73 & 41.60 & $3 \mathrm{C} 430$ & 0.056 & - & - & - & - \\
\hline 3C 197.1 & 0.131 & HEG & 8.1 & 30.30 & - & 3C 436 & 0.215 & HEG & 15.7 & 31.21 & 41.52 \\
\hline 3C 198 & 0.082 & HEG & 18.0 & - & 41.04 & $3 \mathrm{C} 445^{a, b}$ & 0.057 & QSO & 23.5 & 31.34 & 42.05 \\
\hline $3 \mathrm{C} 219$ & 0.174 & WQ & 34.3 & 31.54 & 41.62 & $3 \mathrm{C} 452$ & 0.081 & HEG & 58.3 & 31.24 & - \\
\hline $3 \mathrm{C} 223$ & 0.137 & HEG & 14.2 & 30.57 & 42.17 & $3 \mathrm{C} 456$ & 0.233 & HEG & 14.0 & 31.39 & 42.59 \\
\hline \multirow[t]{2}{*}{ 3C 223.1} & 0.108 & HEG & 8.7 & 30.24 & 41.65 & 3C 458 & 0.289 & - & - & - & - \\
\hline & & & & & & $3 \mathrm{C} 460$ & 0.268 & HEG & 8.1 & 31.39 & 41.67 \\
\hline
\end{tabular}

Redshifts and $S_{\mathrm{t}}$, the total radio flux at $178 \mathrm{MHz}$, are from NED; optical spectral classification is from Jackson \& Rawlings (1997) and C. Willott's web page, http://www-astro.physics.ox. ac.uk/ cjw/ $3 \mathrm{crr} / 3 \mathrm{crr} . \mathrm{html}$;

$\mathrm{L}_{\mathrm{r}}$ is the radio core luminosity at $5 \mathrm{GHz}$ (data are taken from Zirbel \& Baum 1995), except for 3C 123 (Hardcastle et al. 1998, 8.44 GHz), 3C 133 (Nilsson 1998, $5 \mathrm{GHz}$ ), 3C 153 (Hardcastle et al. 1998, 8.44 GHz), 3C 165 (Nilsson 1998, $5 \mathrm{GHz}$ ), 3C 223.1 (Hardcastle et al. 1998, $8.35 \mathrm{GHz}$ ); 3C 349 (Hardcastle et al. 1998, 8.44 GHz). Radio core data at frequencies different from $5 \mathrm{GHz}$ were converted to $5 \mathrm{GHz}$ using a flat $\left(\alpha_{\mathrm{r}}=0\right)$ spectral index. $L_{[\mathrm{OIII}]}$ is the luminosity of the [OIII] line (again from Jackson \& Rawlings 1997 and Willott's web page, except for 3C 88, which has been taken from Tadhunter et al. 1993). (a) line subtracted (see text); (b) saturated core (see Paper II for an outline of the method used to estimate the flux).

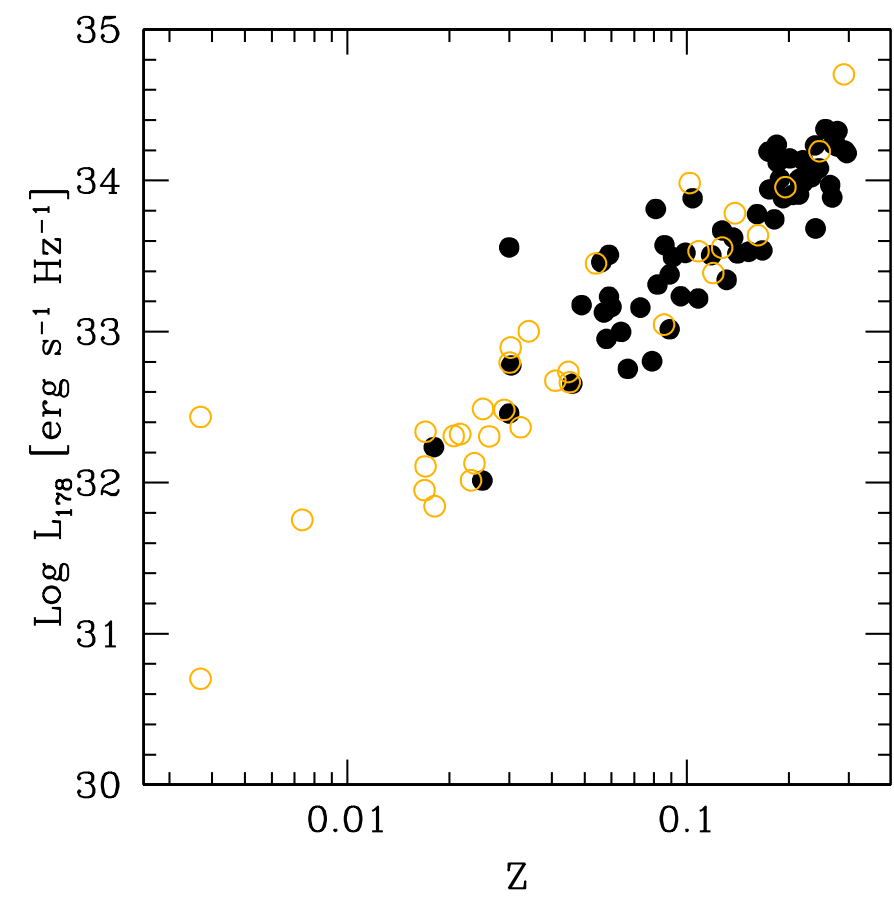

Fig. 1. Total radio luminosity at $178 \mathrm{MHz}$ vs. redshift. FR II are plotted as filled circles, while FR I are empty circles.

cores, CCC). Here we adopt the same definition for CCC as in Papers I and II (a central component with measured $F W H M<0.08^{\prime \prime}$, i.e. unresolved at the HST resolution). In the
Table 2. CCC in FR II radio galaxies.

\begin{tabular}{lccccc}
\hline \hline Class. & CCC & Upper lim. & Complex & Not obs. & Tot. \\
\hline QSO & 4 & 0 & 0 & 0 & 4 \\
WQ & 9 & 0 & 0 & 0 & 9 \\
HEG & 15 & 10 & 3 & 2 & 30 \\
LEG & 4 & 9 & 2 & 1 & 16 \\
Unclass. & 2 & 0 & 3 & 1 & 6 \\
\hline
\end{tabular}

$z<0.3$ sample we find $34 \mathrm{CCC}$ and 19 objects with smooth nuclear profiles and no detected central source. In these objects, we evaluate the upper limit to any central component measuring the light excess of the central $3 \times 3$ pixels with respect to the surrounding galaxy background. In 8 galaxies, the presence of complex morphologies prevents us from evaluating a reliable upper limit to any central source.

Considering each spectral class of FR II separately, we find that all objects classified as QSO and WQ have a CCC; among the narrow-lined objects, 15 HEG (and 4 LEG) have a CCC, for $10 \mathrm{HEG}$ (and $9 \mathrm{LEG}$ ) we are able to set upper limits to the optical nuclear emission, while 3 HEG (and 2 LEG) have complex nuclear morphologies. Of the unclassified sources, 2 have a CCC and 3 have complex nuclei.

We performed aperture photometry of the 34 CCC, using the same technique as described in Paper I. We evaluate the background level at a distance of $\sim 5$ pixels $\left(\sim 0.23^{\prime \prime}\right)$ from the center. The dominant photometric error is thus the determination of the background in regions of steep brightness 
gradients, especially for the faintest cores, resulting in a typical error of $\sim 10 \%$.

All images were taken with broad band filters which, within our redshift range, include emission lines ( $\mathrm{H} \alpha+[\mathrm{N} \mathrm{II}])$. Unfortunately, no HST narrow band images are available for our sources in this spectral range. Since the broad line emission is probably cospatial to the optical CCC, we corrected the broad band fluxes from line contamination in the case of two objects (3C 227 and 3C 445), for which ground based measurements of the broad $\mathrm{H} \beta$ line flux are available (Tadhunter et al. 1993), assuming a ratio $\mathrm{H} \alpha / \mathrm{H} \beta=6$ (Netzer 1990). In these cases, the resulting emission line contribution is $15-20 \%$ of the total flux measured in the F702W filter ${ }^{1}$. We have not corrected the flux of the other QSO and WQ, however, we have tested that the total $\mathrm{H} \alpha$ line flux (Baum \& Heckman 1989) would represent only $10-20 \%$ of the CCC flux. We expect line contribution to be even smaller in the case of HEG and LEG, due to the wide spectral range covered by the filter used $(\sim 2000 \AA)$ with respect to typical narrow line equivalent widths. In Table 3 we report the luminosity of the CCCs for the sample of FR II with $z<0.3$.

Let us now analyze the behavior of FR II nuclei in the plane defined by the radio core luminosity $L_{\mathrm{r}}$ and optical CCC luminosity $L_{0}$ (Fig. 2), similarly to what we have done in Papers I and II and considering their different optical spectral classification.

The nuclei of QSO and WQ (the broad-lined objects) are among the brightest, both in $L_{\mathrm{r}}$ and $L_{\mathrm{O}}$. They are found exclusively in the high luminosity region of the plane, for $L_{\mathrm{r}}>$ $10^{30.4} \mathrm{erg} \mathrm{s}^{-1} \mathrm{~Hz}^{-1}$ and $L_{\mathrm{o}}>10^{28} \mathrm{erg} \mathrm{s}^{-1} \mathrm{~Hz}^{-1}$ ). Most of them show an optical excess of about 2 dex with respect to the radio-optical core correlation of FR I CCC. Conversely, nuclei of LEGs are only found at low luminosities, i.e. for $L_{\mathrm{o}}<$ $10^{27.5} \mathrm{erg} \mathrm{s}^{-1} \mathrm{~Hz}^{-1}$ and $L_{\mathrm{r}}<10^{32} \mathrm{erg} \mathrm{s}^{-1} \mathrm{~Hz}^{-1}$ and all lie close to (or just below) the FR I correlation.

The behavior of HEGs is more complex. Their nuclei span a larger range of luminosity, both in radio and optical. Overall, their CCC (or the upper limits) lie along the FR I correlation, but there are at least two clear exceptions are present (namely 3C 184.1 and 3C 197.1) which are located well above it.

\section{Discussion}

The behavior of optical nuclei of the various sub-classes of FR II in the radio-optical plane for this sample essentially confirms what has been observed in the lower redshift sources discussed in Paper II. However, as the total number of sources here is more than doubled, we can now discuss in more detail what the different position on the radio-optical plane tells us about the nature of the optical nuclei. Note that in the highest redshift sources, the CCC might be contaminated by a contribution from extended features such as the synchrotron jets observed in some of the low redshift sources. However, we are confident that this issue does not significantly alter our results,

\footnotetext{
${ }^{1}$ Other authors (e.g. Heckman et al. 1989) adopt a value of $\mathrm{H} \alpha / \mathrm{H} \beta \sim 2$ for radio galaxies. Therefore our estimate might be considered as an upper limit to the line contamination.
}

Table 3. FR II radiogalaxies nuclei.

\begin{tabular}{|c|c|c|c|}
\hline Source Name & $\begin{array}{c}\log L_{\mathrm{o}} \\
\operatorname{erg~s}^{-1} \mathrm{~Hz}^{-1}\end{array}$ & Source Name & $\begin{array}{c}\log L_{\mathrm{o}} \\
\operatorname{erg~s}^{-1} \mathrm{~Hz}^{-1}\end{array}$ \\
\hline $3 \mathrm{C} 15$ & $<26.91$ & $3 \mathrm{C} 234$ & 29.10 \\
\hline $3 \mathrm{C} 17$ & 28.93 & $3 \mathrm{C} 236$ & $<27.04$ \\
\hline $3 \mathrm{C} 18$ & 28.62 & $3 \mathrm{C} 284$ & complex \\
\hline 3C 33 & not obs. & $3 C 285$ & 25.65 \\
\hline 3C 33.1 & 28.19 & 3C 287.1 & 28.71 \\
\hline $3 \mathrm{C} 35$ & $<26.48$ & 3C 300 & 27.79 \\
\hline $3 \mathrm{C} 40$ & complex & $3 \mathrm{C} 303$ & 28.66 \\
\hline $3 \mathrm{C} 61.1$ & not obs. & 3C 318.1 & $<25.67$ \\
\hline $3 C 63$ & 28.29 & $3 C 319$ & $<27.40$ \\
\hline $3 \mathrm{C} 79$ & 28.33 & 3C 321 & complex \\
\hline $3 C 88$ & 26.60 & 3C 323.1 & 30.14 \\
\hline 3C 98 & $<25.67$ & $3 C 326$ & $<27.09$ \\
\hline 3C 105 & not obs. & $3 \mathrm{C} 327$ & $<26.55$ \\
\hline $3 \mathrm{C} 111$ & 28.14 & 3C 332 & 28.79 \\
\hline $3 \mathrm{C} 123$ & $<26.44$ & 3C 349 & 28.16 \\
\hline $3 \mathrm{C} 132$ & $<26.83$ & $3 \mathrm{C} 353$ & $<25.53$ \\
\hline $3 C 133$ & 28.00 & $3 \mathrm{C} 357$ & $<26.90$ \\
\hline $3 C 135$ & 27.36 & 3C 379.1 & $<27.21$ \\
\hline 3C 136.1 & complex & 3C 381 & $<27.52$ \\
\hline $3 \mathrm{C} 153$ & $<26.66$ & 3C 382 & 29.72 \\
\hline $3 C 165$ & 27.28 & $3 \mathrm{C} 388$ & 27.26 \\
\hline $3 \mathrm{C} 166$ & 28.01 & 3C 390.3 & 29.04 \\
\hline $3 \mathrm{C} 171$ & 26.50 & $3 \mathrm{C} 401$ & 27.62 \\
\hline 3C 173.1 & complex & $3 \mathrm{C} 402$ & 26.59 \\
\hline $3 \mathrm{C} 180$ & complex & $3 \mathrm{C} 403$ & 26.65 \\
\hline 3C 184.1 & 28.17 & $3 \mathrm{C} 430$ & complex \\
\hline $3 \mathrm{C} 192^{a}$ & $<26.99$ & $3 \mathrm{C} 436$ & complex \\
\hline 3C 197.1 & 28.10 & $3 \mathrm{C} 445$ & 29.27 \\
\hline 3C 198 & 28.03 & $3 \mathrm{C} 452$ & $<26.89$ \\
\hline $3 C 219$ & 28.64 & $3 \mathrm{C} 456$ & 28.50 \\
\hline $3 \mathrm{C} 223$ & $<27.27$ & $3 \mathrm{C} 458$ & not obs \\
\hline 3C 223.1 & $<27.18$ & $3 \mathrm{C} 460$ & 27.60 \\
\hline $3 \mathrm{C} 227$ & 28.84 & & \\
\hline
\end{tabular}

All observations are with the $F 702 W$ filter, except (a) for which the $F 555 \mathrm{~W}$ has been used. All luminosities are calculated at $7000 \AA$ (rest frame) and have been $K$-corrected assuming an optical spectral index $\alpha_{\mathrm{o}}=1$.

since these features are observed only in a small fraction of FR I galaxies (possibly only in those with jets pointing towards us). Furthermore, the contribution from the observed jets (e.g. in 3 C 264) is typically less than $20 \%$ of the CCC flux.

\subsection{QSO and WQ: The broad-lined objects}

Let us firstly examine the case of WQ and QSO, the broad-lined objects. As discussed in Paper II, the optical excess shown by these sources, with respect to the radio core emission, can be explained if the dominant component in the optical band is due to thermal emission from an accretion disc.

A very important result here is that the optical nuclei of BLO are present only for $L_{\mathrm{o}}>L_{\mathrm{o} \text {,min }} \sim 10^{28} \mathrm{erg} \mathrm{s}^{-1} \mathrm{~Hz}^{-1}$, corresponding to a luminosity $v L_{v} \gtrsim 4 \times 10^{42} \mathrm{erg} \mathrm{s}^{-1}$. A rather different behavior is seen in radio-quiet AGN, in which broad lines are seen in objects that span many orders of magnitude 


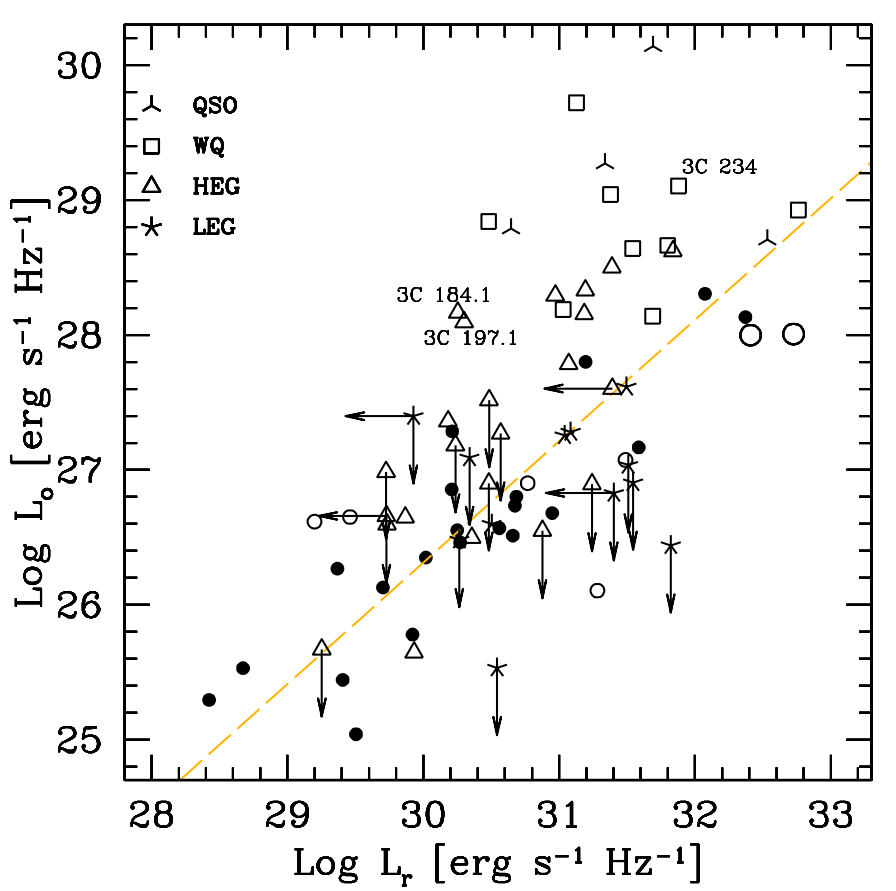

Fig. 2. Optical luminosity of the $\mathrm{CCC}$ vs. radio core luminosity. small circles are FR I (open circles represent upper limits, filled circles are detections); FR II are plotted as different symbols depending on their optical spectral classification (the 2 large open circles represent unclassified objects). With respect to Figs. 5 and 3 of Papers I and II, respectively, we have removed 3C 386 since from new spectroscopic observations of this object it has bees assessed that the optical central source is a star superimposed on the center of the galaxy (Marchesini et al. in prep.).

in nuclear optical luminosity, from LINERS to powerful QSO. Recent works have indeed shown that in LINERS (and in several Seyfert 1 galaxies) the nuclear bolometric luminosity of type 1 radio quiet AGNs can be as low as $L_{\text {bol }} \sim 10^{40}$ (see e.g. Barth et al. 2001 for NGC 4579, Moran et al. 1999 for NGC 4395).

We are quite confident that the lack of BLO below this limit is not due to selection effects. In fact, although spectral data of 3CR radio galaxies are not homogeneous, Willott et al. (2000) have shown that in these low redshift sources the most prominent optical broad line $(\mathrm{H} \alpha)$ should be easily detected if the observed trend between the extended jet and emission line power extends smoothly to the lower luminosity objects (Rawlings \& Saunders 1991; Celotti et al. 1997).

If the optical nucleus observed in BLO is directly related to the inner disk, we can derive some important parameters of the accretion process, under the assumption that the optical luminosity is a fixed fraction of $L_{\mathrm{bol}}$, the bolometric luminosity of the AGN. This translates to $L \gtrsim 3 \times 10^{-4} \eta L_{\mathrm{Edd}}$, where $\eta=L_{\mathrm{bol}} / L_{\mathrm{o}}$ and $L_{\mathrm{Edd}}=1.3 \times 10^{46} M_{8} \mathrm{erg} \mathrm{s}^{-1}$ is the Eddington luminosity for a $10^{8} M_{\odot}$ black hole. For any fixed value of the radiative efficiency $\epsilon$, this in turn translates in an accretion rate $\dot{m} \equiv \dot{M} / \dot{M}_{\mathrm{Edd}} \simeq 3 \times 10^{-4} M_{8}^{-1} \epsilon^{-1} \eta$. Assuming $\epsilon=0.1$, i.e. radiatively efficient accretion, and $\eta \sim 15$, following Elvis et al. (1994), the value of the accretion rate corresponds to $\dot{m} \simeq 5 \times 10^{-2} M_{8}^{-1} \epsilon_{0.1}^{-1} \eta_{15}$. Note, however, that
Zheng et al. (1997) have recently found that the peak of the disk radiated power occurs at a much lower frequency than previously evaluated, and possibly only a factor of 2 higher than the optical luminosity. This implies, for a fixed optical luminosity, a lower value of $L_{\mathrm{bol}}$ and in turn a lower accretion rate.

A possible scenario for the presence of a lower limit in the optical luminosity of BLO is that our estimate of $\dot{m}$ represents a threshold at which the accretion process changes its regime. For lower values of $\dot{m}$ this accretion might occur at a low radiative efficiency, in the form of an advection dominated flow (ADAF, see e.g. Narayan \& Yi 1995), an adiabatic inflow-outflow (ADIOS, Blandford \& Begelman 1999), or a convection-dominated flow (CDAF, Narayan et al. 2000). As shown in Paper I, FR I radio-galaxies, excluding the small minority of them that show clear sign of quasar activity (e.g. 3C 120), might represent examples of such low efficiency processes. The lower amount of ionizing photons available would account for the lack (or at least for the substantial lower intensity) of broad lines in these objects.

However, the presence of an accretion threshold generates a corresponding threshold in luminosity only if there is a relatively narrow range of black hole masses at a fixed radiative efficiency. While a wide range of black hole masses are likely to be harbored in radio-quiet AGNs, we then speculate that black hole masses in radio-loud AGN could be only restricted to a relatively small range, at the higher end of the black hole mass distribution. This seems to be confirmed observationally, as the measured masses in these objects are all concentrated around $10^{9} M_{\odot}$ (e.g. Dunlop et al. 2001; Laor 2000).

\subsection{High excitation galaxies}

In the standard unification picture, narrow-lined FR II are believed to be intrinsically identical to the broad-lined ones, and obscuration of the central regions is assumed to be the only origin of the difference in the emission line properties of the two classes. Note that this is strictly true only in the case of HEG, for which the narrow line properties (luminosity and excitation state) are similar to those of WQ (and QSO). In other words, HEGs are expected to harbor a hidden quasar, while for LEGs this is not strictly required.

The nature of the nuclei of HEG is puzzling. Since most of them lie on the FR I correlation, they might be interpreted, in analogy with FR I, as due to non thermal synchrotron emission from the base of the jet. Of course this appears to be in contrast to the standard unification scenario. However, two narrow-lined objects (3C 184.1 and 3C 197.1) clearly stand out, showing an optical excess of more than 1 dex when compared to sources with similar radio core power. The nature of these objects is unclear: in 3C 184.1 a broad (although very faint) $\mathrm{Pa} \alpha$ line has been detected while no broad components are observed in optical lines (Hill et al. 1996). A possible explanation is that they are moderately absorbed QSO nuclei.

Another puzzling source is 3C 234. Spectropolarimetric studies of this source showed that its polarized spectrum closely matches that of a QSO, with prominent featureless continuum and broad lines (Antonucci 1990; Tran et al. 1995; 
Hurt et al. 1999). This has been interpreted as the result of scattering of light from a hidden QSO nucleus into our line of sight. Nonetheless, its representative point lies on the correlation (within $2 \sigma$ ). We have calculated that the scattered light exactly corresponds to the flux measured from the nuclear component in the HST images of $3 \mathrm{C} 234^{2}$. We can then argue that the nuclear source in 3C 234 is a compact scattering region (which appears as unresolved also in high resolution polarimetric images obtained with the Keck telescope, Cohen 1999). The same argument applies to 3C 109, a FR II radio galaxy with a redshift of $z=0.3056$, just above the distance limit of our sample. Its polarization properties are reminiscent of those of 3C 234 and it was similarly interpreted as a hidden quasar. Also in this case, we have tested that the scattered continuum matches its CCC flux ${ }^{3}$. Remarkably, its CCC also lies on the correlation (again within $2 \sigma$ ).

Therefore, besides synchrotron radiation from the jet, another possible scenario for the nature of such nuclei is that the observed optical emission might originate from the expected (obscured) quasar component, which is scattered to our line of sight by "mirrors" placed out of the obscuring torus.

From this analysis it turns out that it is very difficult to definitively address the nature of HEG nuclei by only considering their position in the optical-radio plane, particularly in the region of high radio core luminosity, where the FR I correlation intersects the BLO region. In order to clarify their nature, more information is needed. As we show in the following section, a fundamental advancement will be achieved with the inclusion in our analysis of a further parameter, i.e. the luminosity of their emission lines.

\subsection{EW of [OIII] vs. the radio optical correlation: A new diagnostic plane for radio galaxies}

In Fig. 3 we plot the ratio between the [O III] flux and the optical flux of the CCC, which essentially represents the equivalent width of the emission line with respect to the nuclear component, versus the radio core - CCC ratio. This emission line appears in fact to be strictly connected to the nuclear ionizing source. The value of the [OIII] flux is taken by Jackson \& Rawlings (1997) and from the data collected by Chris Willott in his home page (http://www-astro.physics.ox.ac.uk/ cjw/3crr/3crr.html). We have collected [O III] line fluxes for 42 FRII and for 5 FR I, while complete information (line flux, radio and optical core, either detections or upper limits) is available for 33 FR II

\footnotetext{
2 The polarization level of its broad $\mathrm{H} \alpha$ line is $\sim 20 \%$, after removal of the host galaxy starlight. This indicates that the total flux of the scattered $\mathrm{H} \alpha$ is approximately 5 times larger than its polarized component. Applying the same correction to the featureless continuum emission level we infer a scattered flux of $\sim 10^{-16} \mathrm{erg} \mathrm{s}^{-1} \mathrm{~cm}^{-2} \mathrm{~A}^{-1}$. The CCC flux in the HST image of $3 \mathrm{C} 234$ is $1.1 \times 10^{-16} \mathrm{erg} \mathrm{s}^{-1} \mathrm{~cm}^{-2} \mathrm{~A}^{-1}$.

3 The continuum flux in 3C 109 is as polarized as the broad lines (indicating the absence of a significant diluting component) at a level of $\sim 7 \%$. Given a polarized flux at $7000 \AA$ of $\sim 2 \times$ $10^{-17} \mathrm{erg} \mathrm{s}^{-1} \mathrm{~cm}^{-2} \mathrm{~A}^{-1}$ this translates into a scattered component of $\sim 3 \times 10^{-16} \mathrm{erg} \mathrm{s}^{-1} \mathrm{~cm}^{-2} \mathrm{~A}^{-1}$. The nuclear source in the HST images has a flux of $2.8 \times 10^{-16} \mathrm{erg} \mathrm{s}^{-1} \mathrm{~cm}^{-2} \mathrm{~A}^{-1}$.
}

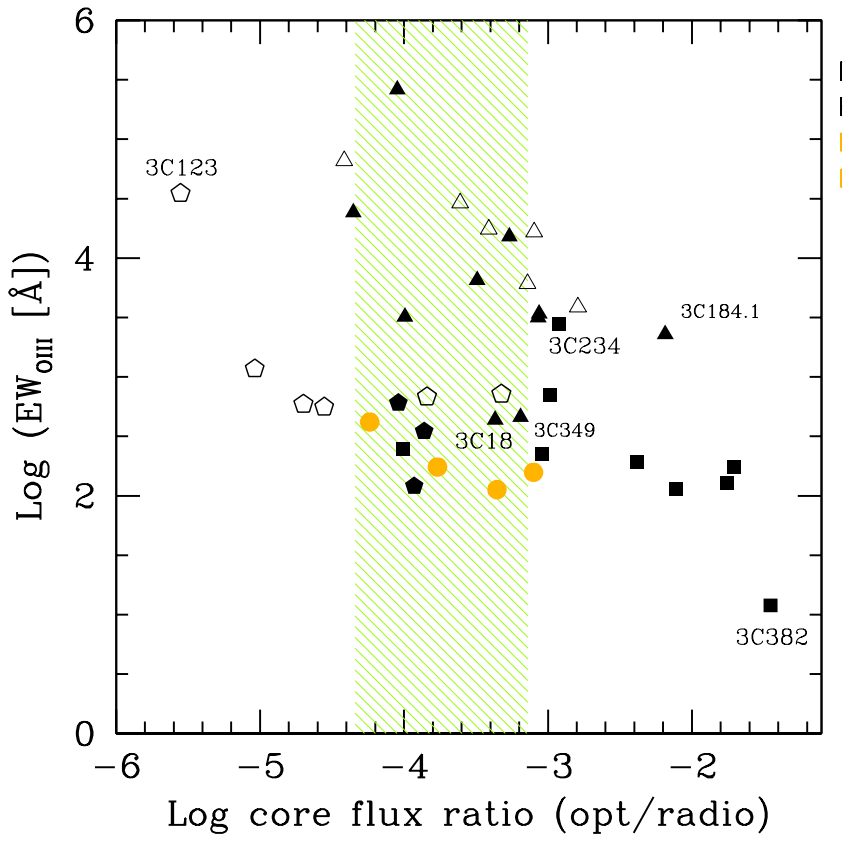

Fig. 3. Equivalent width of the [OIII] emission line, measured with respect to the CCC emission, is plotted vs. the ratio between the optical CCC to radio core flux. Filled symbols represent CCC detections, while empty symbols are upper limits. Squares are BLO, triangles are HEG, pentagons are LEG and circles are FR I. The shaded area represents the dispersion $(1 \sigma)$ of the linear correlation between radio core and CCC luminosity found for FR I.

and 4 FR I. The location with respect to the $\mathrm{X}$ axis is readily understood noting that the radio-optical core correlation of FR I is represented by a fixed radio-to-optical ratio, i.e. a vertical stripe in this plane, centered on $F_{\mathrm{o}} / F_{\mathrm{r}} \sim 10^{-3.7}$. The $1 \sigma$ region around this value is represented in the figure as the shaded area. Objects lying on the right side of the plot show an optical excess.

In this plane, sources separate in two regions corresponding to equivalent width values $E W \sim 10^{2.5} \AA$ or $E W>10^{3.5} \AA$. In the first region we find all QSO and WQ (squares) ${ }^{4}$ and LEG (pentagons). Interestingly, also the four FR I (3C 66B, 3C 84, 3C 346 and 3C 449, plotted as filled grey circles) for which these data are available fall into this region. Conversely, all but two of the HEG have much larger equivalent widths, from $E W \sim 10^{3.5} \AA$ to $E W \sim 10^{5.5} \AA$.

The separation of different sources depending on the equivalent width of their emission line is readily expected from the unified models. This is due to the effects of obscuration that strongly reduces the observed nuclear continuum, while the line emission, produced at larger distance from the nucleus, is unaffected. We can then argue that all sources with $E W>1000 \AA$ are hidden $\mathrm{BLO}^{5}$. In sources with very high values of $E W\left(E W>10^{3} \AA\right)$, a strong ionization source,

\footnotetext{
${ }^{4}$ The only BLO with value of $E W$ higher than $1000 \AA$ is $3 \mathrm{C} 234$ which, as already pointed out in Sect. 2 , should be more properly re-classified as a HEG.

5 Note that since the [OIII] emission line regions have a typical dimension of $10 \mathrm{kpc}$ (e.g. Baum \& Heckman 1989), such high values of the $E W$ are still compatible with the assumption made in Sect. 3 of a small line contamination for the nuclear flux.
} 


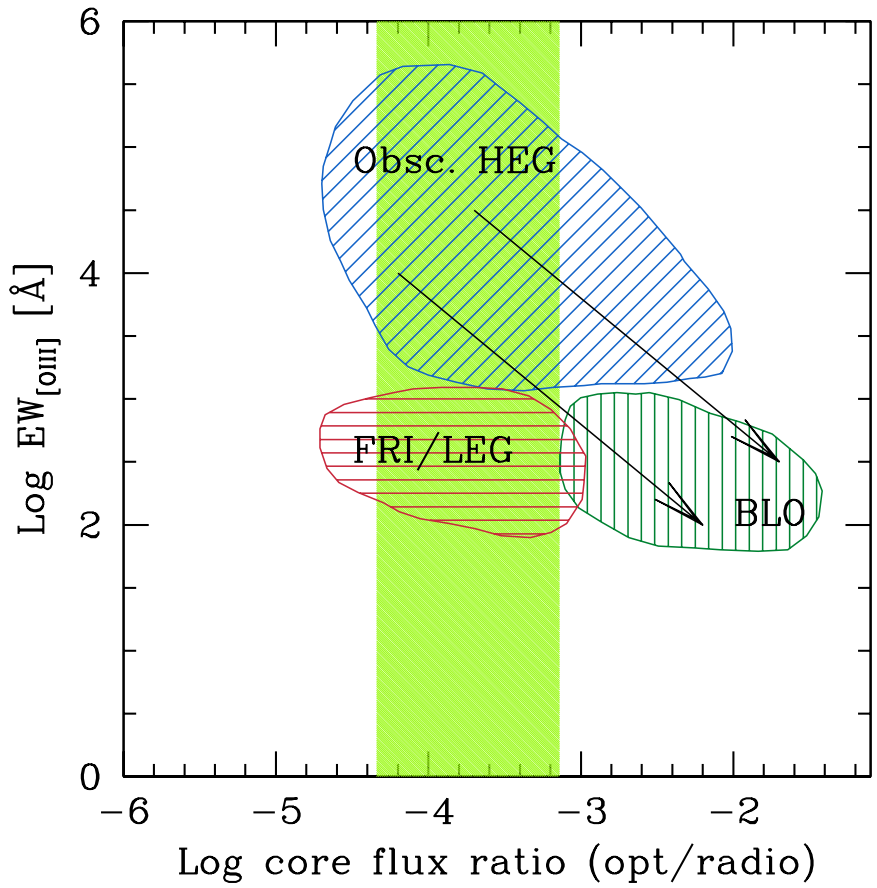

Fig. 4. Different classes of radio galaxies occupy different regions in their "fundamental" diagnostic plane, made by the $E W$ of the [OIII] line plotted vs. the optical to radio core flux ratio. "Obscured" HEG have the highest $E W$. These sources can be lead back to the region of BLO if their optical flux is increased by a factor of $\sim 100$ (arrows).

obscured to our viewing angle, must be present. This argument follows essentially the same line of reasoning that has been previously applied to Seyfert galaxies for which, in several cases, a deficit of ionizing photons with respect to their line luminosity has been found (e.g. Kinney et al. 1991). Assuming the median value of the $E W \sim 10^{2.5} \AA$ for BLO, the spread in the $E W$ of HEG corresponds to a scattering fraction varying between 0.001 and 0.03 . Indeed note that obscuration, which affects only the optical luminosity, moves the objects along straight lines parallel to the diagonal of the plane. Indeed, all galaxies with high values of $E W$ can be led back to the region typical of QSO increasing their nuclear luminosity by, on average, a factor of $\sim 100$ (see Fig. 4).

The sources with low values of $E W$ are all clustered around $E W \sim 300 \AA$. This value is essentially independent of the source class of luminosity. A low value of $E W$ thus appears to be the signature that we are directly observing the source of ionization. While this is straightforward for QSO and WQ, it implies that in FR I either the source of gas ionization is the synchrotron emission from the jet, or any other contribution must have an intensity comparable to the jet emission. Since this must hold for all radio luminosities, the latter hypothesis appears to be quite finely tuned. The correlation found by Verdoes Kleijn et al. (2001) between the nuclear $\mathrm{H} \alpha$ luminosity and the optical CCC luminosity in FR I further supports the former scenario.

Only two HEG lie among the "unobscured" sources: interestingly, these galaxies (3C 18 and 3C 349) also lie on the FR I correlation, on its high luminosity end. Therefore, due to their position in both planes, we can interpret that these galaxies are true unobscured and synchrotron dominated "FR I-like" FR II.

Since this plane allow us to separate and to identify the nature of the nuclear sources of both FR I and FR II, we consider this as a new "fundamental" diagnostic plane for radio galaxies.

Another interesting consequence of this new characterization is the possibility of inferring the nature of the sources in which the nucleus is not detected. Upper limits among the HEG would be attributed to obscuration of the central quasar-like source and small amount of scattered radiation (compared to the central surface brightness of the host galaxy). In the case of LEG, this issue is more complex: as we expect their nuclei to be fainter, the ambiguity between low contrast and even mild absorption holds. However, in case of absorption by a toruslike structure in LEG, objects with upper limits and detections should have a different orientation distribution. In order to address this issue, and before proceeding to discuss the properties of LEG and their role in the unification models, it is necessary to analyze how the different classes relate to the radio properties.

\subsection{Relationship with the radio properties}

In this section we compare the distribution of the different subclasses of FR II both in total radio luminosity at $178 \mathrm{MHz}$ and in $R$, the ratio between core and total radio power, which is often used as an orientation indicator. This is crucial both to understand the role of the different sources in the unification scheme, and to know whether orientation plays a role in the presence (or absence) of the CCC.

Although a similar analysis has been already done on a wider sample of 3CR sources by Laing et al. (1994), it is very important to perform statistical tests on our sample, taking also into account the newly obtained information on the optical nuclear properties of our objects. In order to improve the statistics, we have considered WQ and QSO as a single class, due to their similar properties. However, we have checked that this does not affect the results, as the objects belonging to these two classes also share the same range in both $L_{178}$ and $R$. In Fig. 5 we show the distribution in $L_{178}$ for the three different spectral classes. Although the number of objects for each class is limited, it is straightforward to note that: i) they share the same range in total power; ii) they are similarly distributed. In particular, this implies that within our range of redshift the three subclasses of FR II co-exist and cannot be discriminated on the basis of their total radio luminosity.

We use a Kolmogorov-Smirnov test to see whether the distributions of $R$ for the various sub-classes, as well as for objects with different nuclear properties, are statistically different. From this analysis, we deduce the following:

- broad-lined objects have higher $R$ with respect to both HEG $(P>99.96 \%)$ and LEG $(P>88 \%)$. This fits in the framework in which BLO are sources where the nuclear emission is seen directly, and the observing angle is smaller than the HEG one (but still larger than in beamed blazar sources). Their high 

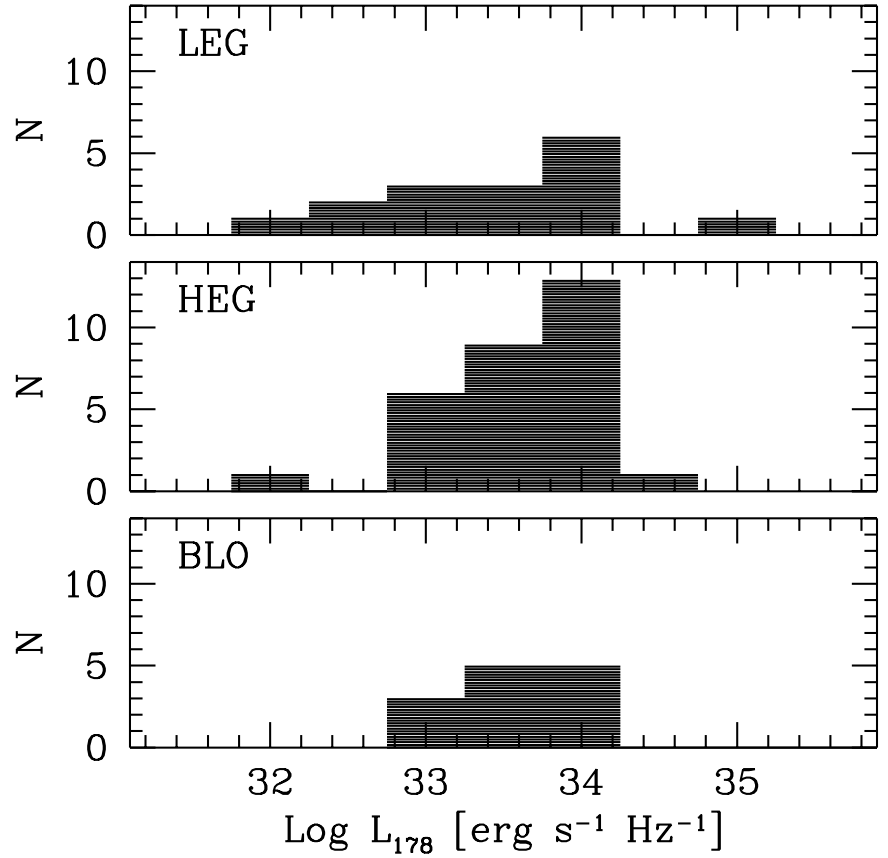

Fig. 5. Histograms of the radio total luminosity at $178 \mathrm{MHz}$ for the three spectral classes of FR II.

luminosity CCCs can be produced in the very inner regions of the AGN, possibly in the accretion disc;

- the distributions in $R$ of HEG and LEG are different $(P>$ 91.9\%). This is consistent with the conclusions of Laing et al. (1994) that LEG constitute a randomly oriented sample;

- an important result here is that HEG and LEG with and without CCC do not have different distributions in $R$. This might imply that the presence of CCC in these sources does not depend strongly on orientation. Note that this is quite obvious for HEG, if the origin of the majority of their nuclei is scattered radiation.

In light of these results, we have acquired enough information to discuss the properties of LEG.

\subsection{The low excitation galaxies}

As already pointed out above, the nuclei of LEG, when detected, lie on the FR I correlation, and they also have $E W$ very close to those of FR I. From the above statistical analysis, we also confirm that LEGs in our sample have a broad distribution in $R$, compatible with that of a randomly oriented population. Therefore, from the point of view of their radio-optical nuclear properties, these objects are indistinguishable from FR I. Since their nuclei appear to be dominated by synchrotron radiation from the relativistic jet, the lack of strong (and high ionization) emission lines in their spectra might be accounted for by a lower number of ionizing photons.

These objects, when observed along the jet axis, should appear as BL Lac objects and can therefore account for the known BL Lacs with FR II radio morphology. Note that this is in contrast with a "standard" unification model, which associates all FR II to quasars, but these objects can well account for BL Lacs

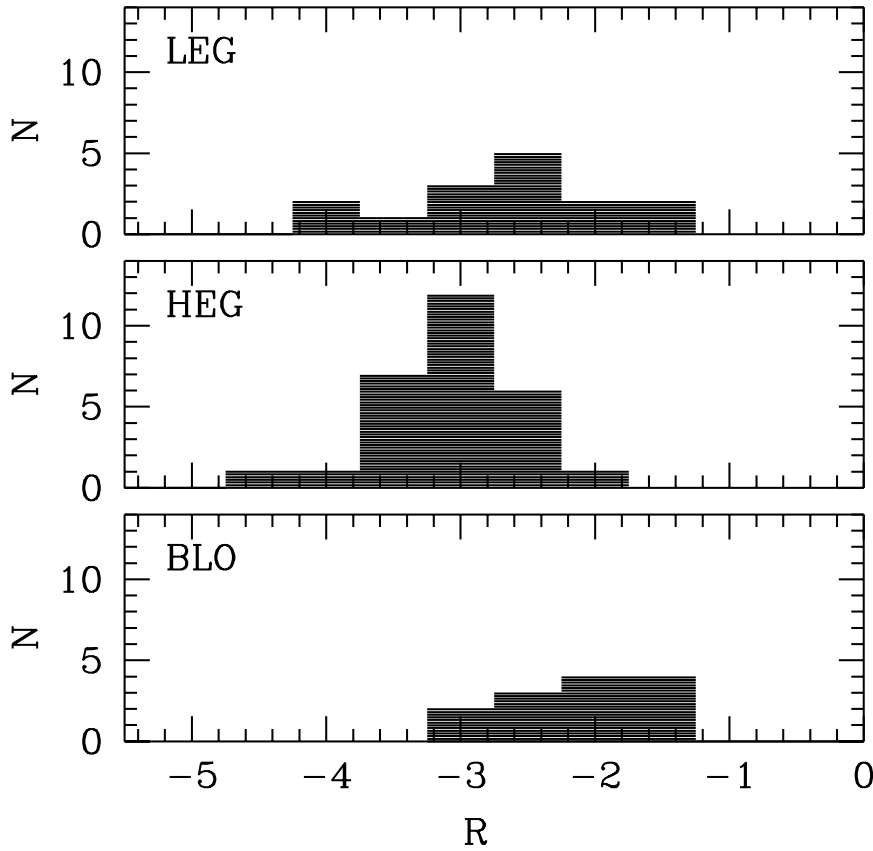

Fig. 6. Histograms of the radio core (at $5 \mathrm{GHz}$ ) to total flux (at $178 \mathrm{MHz}$ ) for the three spectral classes of FR II.

with both an extended radio morphology (e.g. Kollgaard et al. 1992) and extended radio power (Cassaro et al. 1999) typical of FR II. Jackson \& Wall (1999) have also shown that both the evolution and unification of FR I and FR II with BL Lacs and flat-spectrum quasars (FSRQ) are well accounted for by a dual-population scheme, which considers FR I and LEG as a single population, associated with BL Lacs, while all other FR II are unified to FSRQ. Thus, our results are in agreement with such a scenario.

Some independent pieces of evidence appear to confirm this picture. Recently, Willott et al. (2000) have found that the fraction of quasars in the 3CRR, 6C and 7C samples (having excluded objects with FR I radio morphology) decreases as luminosity (both radio and narrow line) decreases; this behavior might be explained in the framework of a "two population" model: at low luminosities, a significant fraction of sources are "starved quasars", and their nuclear properties, as well as the characteristics of their spectra, are similar to those of FR I. It is therefore tempting to identify this class with LEGs.

We must point out that a particular source (3C 123) does not appear to fit this scenario, since it has no optical CCC and its equivalent width of [OIII] is higher than $10^{3}$ (see Fig. 3). However, its $E W$ is larger by more than one order of magnitude compared to all of the other LEG, and also its O[III] luminosity is more typical of HEG. Unfortunately, no detailed spectral information is available in the literature for this object. In particular, the $[\mathrm{OIII}] /[\mathrm{OII}]$ ratio, which is a fundamental parameter for a correct classification, is unknown. Therefore, in light of its position in the diagnostic plane, we argue that $3 \mathrm{C} 123$ should be re-classified as a HEG. 


\section{Summary and conclusions}

We have analyzed the optical nuclear properties of a complete sample of 65 FR II radio galaxies up to $z=0.3$ from the $3 \mathrm{CR}$ catalog. The overall scenario basically confirms the findings for the lower redshift sample $(z<0.1)$ presented in Chiaberge et al. (2000). However, the larger number of sources (more than double) allows us to reveal a richer and more complex behavior, which turns out to be closely associated with the optical spectral properties of the different objects. The nuclear properties of FR II, as inferred from our analysis, can be summarized as follows.

While the great majority of FR I radio galaxies (optical and radio) nuclei are dominated by non-thermal synchrotron emission from the relativistic jet, the FR II population is not homogeneous. Although optical Central Compact Cores appear to be a common feature also in FR II galaxies, their origin can be ascribed to different physical processes.

BLO typically have the brightest nuclei which show a large optical excess with respect to the radio-optical correlation found for FR I. This is readily explained if the dominant component in the optical band is due to thermal emission from an accretion disc. We found that optical nuclei of BLO are present only for $L_{\mathrm{o}}>10^{28} \mathrm{erg} \mathrm{s}^{-1} \mathrm{~Hz}^{-1}$. A rather different behavior is seen in radio-quiet AGN, in which broad lines are seen in objects that span many orders of magnitude in nuclear optical luminosity, from LINERS to powerful QSO. As we argued that this effect cannot be ascribed to obscuration or selection effects, we suggest that this is the manifestation of a threshold in the efficiency of the accretion process, from the standard optically thick, geometrically thin accretion disk to low radiative accretion flows.

Note that the presence of any limit in luminosity requires a well defined behavior of the accretion rate and radiative efficiency but also a narrow distribution in black hole masses for radio-loud AGNs. This conjecture seems indeed to be supported by the direct measurements available to date.

The nature of the nuclei of HEG is certainly more complex. Although most sources lie along the FR I correlation, there are at least two clear exceptions, showing a significant optical excess. Furthermore, there are at least two sources (3C 234 and 3C 109) for which spectropolarimetric studies clearly showed that they harbor a hidden QSO nucleus. The amount of scattered continuum light matches exactly the flux of their nuclear component in the HST images, indicating that their CCC is a compact scattering region. Nonetheless, their representative points would lie on the correlation by coincidence. From this analysis it turns out that in general it is impossible to definitively address the nature of HEG nuclei by only considering their position in the optical-radio plane. A fundamental advance is achieved with the inclusion in our analysis of a further parameter, i.e. the luminosity of their emission lines.

In the plane formed by the "nuclear" $E W$ of the [OIII] line vs. the optical excess with respect to the non-thermal jet emission (a new "fundamental" diagnostic plane?), the different classes of sources clearly separate according to the nature of their nuclei. For low $E W$ values $\left(\sim 10^{2.5} \AA\right)$ we find all QSO, WQ, LEG and FR I, which differ only by the amount of the optical excess. On the other hand, all but two of the HEG have much larger equivalent widths $\gtrsim 10^{3.5} \AA$. The separation of sources depending on their line equivalent width is indeed expected from the unified models, as obscuration reduces the nuclear continuum emission while the line emission is less affected or unaffected. In sources with very high values of $E W$ a strong ionization source, obscured to our viewing angle, must be present. We can then argue that all sources with high $E W$ are hidden BLO.

The low $E W$ region would then contains objects in which we see directly the source of ionization. A ramification of this result is that in FR I the most likely dominant source of gas ionization is synchrotron emission from the jet.

Only two HEG are located among the "unobscured" sources: interestingly, these galaxies (3C 18 and 3C 349) also lie on the FR I correlation. Therefore, due to their position in both planes, we identify these objects with true unobscured "FR I-like" FR II.

According to the scenario proposed here, the non-detection of CCC in galaxies of different classes should have different origins. For LEG this might be due to either a low contrast with the stellar host galaxy emission or to a (moderate) amount of absorption, randomly oriented with respect to the jet. On the other hand, for HEG this can be only attributed to a lower amount of scattered nuclear radiation.

The picture which emerges is that radio galaxies manifest in two types, which are not directly related to the extended FR I/FR II dicothomy. In the framework of the unification scheme, BLO and obscured HEG appear to have the same nuclear structure: intense thermal disk (ionizing) emission, substantial broad emission line region, torus-like absorber and, of course, powerful jets. On the other hand, LEG, FR I and unabsorbed HEG constitute a distinct population, characterized by low radiative efficient accretion, weak or absent broad line emission, lack of a significant nuclear absorbing structure. Unfortunately we have complete information (radio, optical and emission line) for only roughly half of the sources in our sample. Although we can only make the assumption that there is no bias in their selection, we can estimate that the population of FR II is composed of $\sim 50 \%$ obscured sources harboring a quasar nucleus, $25 \%$ BLO, 20\% LEG and $\sim 5 \%$ FR I-like HEG (although so far this is assessed only for two sources). The latter two classes can account for BL Lac objects with FR II radio morphology and extended radio power (e.g. Kollgaard et al. 1992; Cassaro et al. 1999). This scenario apparently poses problems for the simplest unification models, in particular in identifying the beamed counterparts of FR I-like HEG. However, strong and high excitation narrow emission lines are indeed observed in few BL Lacs (Landt et al. 2001). Moreover, the line equivalent width in the beamed objects will be reduced by a factor $\sim 10^{4}$ (the typical ratio between the nuclear optical luminosity between radio galaxies and BL Lacs, see Capetti et al. 2002) producing values consistent with a BL Lac classification.

A possible way to test the overall picture is to look at the spectral properties of the nuclei. BLO and scattered nuclei are expected to be different from the FR I and FR I-like synchrotron ones: in particular, we expect to observe flatter 
spectral indices, typical of quasars, in BLO, indicating the presence of a thermal blue bump. Due to the large uncertainties, optical observations (even in two different bands) are not enough to determine the spectral slope, which instead could be better measured by taking advantage of the UV information (Chiaberge et al. 2002). In addition, an infrared nuclear excess is expected in the obscured radio galaxies, while this has to be absent in FR I-like objects, as promisingly shown by Whysong \& Antonucci (2001) for the case of 3C 405 (a true obscured quasar) and 3C 274 (M 87). A further diagnostic tool is of course the observations in the X-ray band, which can reveal the presence of different amounts of nuclear absorption.

Finally, it would be particularly interesting to analyze how the properties of the newly discovered quasars showing FR I radio morphology (Blundell \& Rawlings 2001; Lara et al. 1999) fit in our picture. In a sense they might represent the analogous (but oposite) case of LEG, where an FR II harbors an FR I nucleus. The spectral information available in the literature for such objects is, to our best knowledge, not yet sufficient to state where these sources are located in the diagnostic plane of Fig. 3. However, if they are indeed broad-lined FR I (showing optical thermal emission from the accretion disk) they should be placed in the lower-right end of the plane, among the BLO. If this is the case, the properties of these peculiar sources might bring further support to the models that claim that the nuclear structure is not directly connected to the extended radio morphology (e.g. Bicknell 1984, 1994; Gopal-Krishna \& Wiita 2001).

Acknowledgements. The authors thanks F. D. Macchetto for insightful discussions and Robert Antonucci for useful comments. This research has made use of the NASA/IPAC Extragalactic Database (NED) which is operated by the Jet Propulsion Laboratory, California Institute of Technology, under contract with the National Aeronautics and Space Administration.

M.C. wish to thank S. Crawshaw, B. Waghorn and A. De Martino for stimulating conversations.

\section{References}

Antonucci, R., \& Barvainis, R. 1990, ApJ, 363, L17

Barth, A. J., Ho, L. C., Filippenko, A. V., Rix, H., \& Sargent, W. L. W. 2001, ApJ, 546, 205

Barthel, P. D. 1989, ApJ, 336, 606

Baum, S. A., \& Heckman, T. 1989, ApJ, 336, 681

Bicknell, G. V. 1994, ApJ, 422, 542

Bicknell, G. V. 1984, ApJ, 286, 68

Biretta, J. A., Burrows, C. J., Holtzman, J. A., et al. 1996, Wide Field and Planetary Camera 2 Instrument handbook, ed. J.A. Biretta (Baltimore:STScI)

Blandford, R. D., \& Begelman, M. C. 1999, MNRAS, 303, L1

Blundell, K. M., \& Rawlings, S. 2001, ApJ, 562, L5

Cassaro, P., Stanghellini, C., Bondi, M., et al. 1999, A\&AS, 139, 601

Capetti, A., Celotti, A., Chiaberge, M., et al. 2002, A\&A 383, 104

Celotti, A., Padovani, P., \& Ghisellini, G. 1997, MNRAS, 286, 415

Chiaberge, M., Macchetto, F. D., Sparks, W. B., et al. 2002, ApJ, 571, 247

Chiaberge, M., Capetti, A., \& Celotti, A. 2000, A\&A, 355, 873
Chiaberge, M., Capetti, A., \& Celotti, A. 1999, A\&A, 349, 77

Cohen, M. H., Ogle, P. M., Tran, H. D., Goodrich, R. W., \& Miller, J. S. 1999, AJ, 118, 1963

De Koff, S., Baum, S. A., Sparks, W. B., et al. 1996, ApJS, 107, 621

Dunlop, J. S., McLure, R. J., Kukula, M. J., et al. 2001 [astro-ph/0108397]

Elvis, M., et al. 1994, ApJS, 95, 1

Fanaroff, B. L., \& Riley, J. M. 1974, MNRAS, 167, 31

Gopal-Krishna, \& Wiita, P. J. 2001, A\&A, 373, 100

Hill, G. J., Goodrich, R. W., \& Depoy, D. L. 1996, ApJ, 462, 163

Heckman, T. M., Baum, S. A., van Breugel, W. J. M., \& McCarthy, P. 1989, ApJ, 338, 48

Hurt, T., Antonucci, R., Cohen, R., Kinney, A., \& Krolik, J. 1999, ApJ, 514,579

Jackson, C. A., \& Wall, J. V. 1999, MNRAS, 304, 160

Jackson, N., \& Rawlings, S. 1997, MNRAS, 286, 241

Hardcastle, M. J., Alexander, P., Pooley, G. G., \& Riley, J. M. 1998, MNRAS, 296, 445

Kinney, A. L., Antonucci, R. R. J., Ward, M. J., Wilson, A. S., \& Whittle, M. 1991, ApJ, 377, 100

Kollgaard, R. I., Wardle, J. F. C., Roberts, D. H., \& Gabuzda, D. C. 1992, AJ, 104, 1687

Landt, H., Padovani, P., Perlman, E. S., et al. 2001, MNRAS, 323, 757

Laing, R. A., Jenkins, C. R., Wall, J. V., \& Unger, S. W. 1994, The First Stromlo Symposium: The Physics of Active Galaxies, ed. G. V. Bicknell, M. A. Dopita, \& P. J. Quinn, ASP Conf. Ser., 54, 201 Laor, A. 2000, ApJ, 543, L111

Lara, L., Márquez, I., Cotton, W. D., et al. 1999, New Astron. Rev. 43, 643

Ledlow, M. J., \& Owen, F. N. 1996, AJ, 112, 9

Martel, A. R., Baum, S. A., Sparks, W. B., et al. 1999, ApJS, 122, 81

Moran, E. C., Filippenko, A. V., Ho, L. C., et al. 1999, PASP, 111, 801

Narayan, R., \& Yi, I. 1995, ApJ, 444, 231

Narayan, R., Igumenshchev, I. V., \& Abramowicz, M. A. 2000, ApJ, 539, 798

Netzer, H. 1990, in Active galactic nuclei, ed. R. D. Blandford, H. Netzer, L. Woltjer, T. Courvoisier, \& M. Mayor (Berlin; New York: Springer-Verlag)

Nilsson, K. 1998, A\&AS, 132, 31

Orr, M. J. L., \& Browne, I. W. A. 1982, MNRAS, 200, 1067

Owen, F. N. 1993, in Jets in extragalactic radio sources, ed. H. J. Roeser, \& K. Meisenheimer (Springer, New York), 273

Rawlings, S., \& Saunders, R. 1991, Nature, 349, 138

Spinrad, H., Djorgovski, S., Marr, J., \& Aguilar, L. 1985, PASP 97, 932

Tadhunter, C. N., Morganti, R., di Serego-Alighieri, S., Fosbury, R. A. E., \& Danziger, I. J. 1993, MNRAS, 263, 999

Tadhunter, C. N., Morganti, R., di Serego-Alighieri, S., Fosbury, R. A. E., \& Danziger, I. J. 1993, MNRAS, 263, 999

Tran, H. D., Cohen, M. H., \& Goodrich, R. W. 1995, AJ, 110, 2597

Urry, C. M., Padovani, P. 1995, PASP, 107, 803

Verdoes Kleijn, G. A., Baum, S. A., de Zeeuw, P. T., \& O’Dea, C. P. 2001, submitted

Wall, J. V., \& Jackson, C. A. 1997, MNRAS, 290, L17

Whysong, D., \& Antonucci, R. 2001 [astro-ph/0106381]

Willott, C. J., Rawlings, S., Blundell, K. M., \& Lacy, M. 2000, MNRAS, 316, 449

Zheng, W., Kriss, G. A., Telfer, R. C., Grimes, J. P., \& Davidsen, A. F. 1997, ApJ, 475, 469

Zirbel, E. L. 1997, ApJ, 476, 489

Zirbel, E. L. 1996, ApJ, 473, 713

Zirbel, E. L., \& Baum, S. A. 1995, ApJ, 448, 521 